\title{
Surviving the desk-review
}

\author{
Fabian Jintae Froese ${ }^{1} \cdot$ Katharina Bader ${ }^{2}$
}

Published online: 5 February 2019

(c) Springer Nature Limited 2019

One major goal of scientific research is communication with other researchers via publishing the results of your work in scientific journals. Unfortunately, not all manuscripts can be published in esteemed peer-reviewed journals. In fact, many submitted manuscripts will not even enter the peer-review process; they will be deskrejected without receiving detailed feedback from peer-reviewers. What are the reasons for desk-rejects, and what can authors do to avoid them?

Scientific journals receive an increasing number of submissions. For instance, Asian Business \& Management (ABM) received 712 submissions in 2018. To guarantee academic rigor and to identify potentially impactful research, each published manuscript undergoes a thorough peer-review process, involving at least two independent double-blind peer-reviews and an assessment of an editor. However, before manuscripts are introduced into this work-intensive process, they need to pass an in-depth desk-review. This is for an important reason: the peer-review system thrives on voluntary contributions. Reviewing for journals is an important task, and it takes expertise, time and energy from those involved. Therefore, we would like to express our sincere gratitude to all our editors and reviewers who have dedicated their precious time to helping others improve their work. They have done an excellent job in supporting the journal. As the time at the disposal of our editors and reviewers is limited, it is impossible to send all manuscripts into the review process. Consequently, we can only send those manuscripts into the review process, which we believe can have a realistic chance for eventual publication. Therefore, what can authors do to increase the chances of their manuscript surviving desk-review?

In the following, we briefly explain the desk-review process, common mistakes, and provide some recommendations on how authors can avoid such mistakes and thus hopefully have their manuscripts published in ABM.

Fabian Jintae Froese

ffroese@uni-goettingen.de

1 University of Goettingen, Platz der Goettinger Sieben 5, 37085 Goettingen, Germany

2 University of Northumbria, Newcastle-upon-Tyne, England, UK 


\section{The desk-review process}

All incoming submissions to ABM will be screened by an editorial assistant, the reviewing editor and the editor-in-chief. First, the editorial assistant checks formality and runs all manuscripts through a computerized plagiarism detection software. Second, the reviewing editor checks the results of the editorial assistant, reads the manuscript and makes a recommendation on whether to advance the manuscript into the review process or to desk-reject it. Third, the editor-in-chief receives the recommendation from the reviewing editor and scrutinizes the submission. If the recommendation is unequivocal, the editor-in-chief will then either forward the manuscript to a handling editor, e.g., an associate editor or guest editor, or desk-reject it. In case of doubt, the editor-in-chief carefully reads the manuscript to render a decision whether to advance or desk-reject the manuscript. Finally, the handling editor, usually an expert in the area of the manuscript, enters the manuscript into the doubleblind peer-review process or desk-rejects it.

\section{Reasons for desk-rejects}

Unfortunately, because of the high number of submissions, we are not always able to give detailed and comprehensive reasons for all desk-reject decisions. Yet, having received and reviewed hundreds of submissions, there are a couple of general issues that lead to a desk-reject which we would like to share with potential authors. In the following, we will review the common reasons for desk-reject decisions and provide recommendations on how authors can avoid these mistakes. We organize the main reasons into four overarching categories: mismatch with the journal, theoretical contribution, writing, and methods.

\section{Mismatch with the journal}

Each journal has a different mission, topic and methods coverage. ABM covers business and management topics in the areas of corporate governance, entrepreneurship, human-resource management, innovation management, international business, marketing, organizational behavior, organization theory, strategy, and related areas; but desk-rejects articles in other areas that are not within the scope of the journal, e.g., accounting, economics, finance, information systems, operations research. As a region-focused journal, ABM publishes articles that illuminate business and management in and/or from Asia. ABM is open to various methods, including quantitative, qualitative, and conceptual work, and welcomes innovative research methods. For more detailed information about the scope of ABM, see the editorial by the editor-in-chief (Froese 2018): https://link.springer.com/article/10.1057/s4129 1-017-0028-0.

It is important to submit manuscripts to a journal that is interested in your field of research. For instance, if your manuscript is not within the scope of ABM as stated 
above, it will, unfortunately, be automatically desk-rejected. Therefore, we recommend carefully reading the editorial (Froese 2018), browsing ABM's homepage, and prior issues to determine the fit with the journal, to make modifications, or to submit to a different journal with a better fit to avoid mismatch. Furthermore, even though the regional focus of ABM is already clearly reflected in its title, some submissions surprisingly fail to acknowledge the Asian context. The editorial (Froese 2018) describes different ways in which authors could consider the Asian context. Please ensure reviewing this critical aspect carefully before submitting to ABM and consider how best to include the Asian context in your work.

\section{Theoretical contribution}

The potential theoretical contribution of an article is one of the most important criteria to determine the value of a submission. ABM and most other journals emphasize theoretical contributions, and value practical and empirical contributions to a lesser extent. Unfortunately, in a substantial number of submissions, the theoretical contribution is not clear, or only very minor; it seems that sometimes authors themselves are not aware of their theoretical contributions or fail to develop and discuss the novel theoretical insights of their work. Furthermore, a substantial number of submissions do not refer to any theory but merely mention theories without proper integration. Another problem is the replication of prior work without adding any novel insights. Not discarding the value of replication studies, ABM is primarily interested in novel contributions.

In order to highlight the theoretical contribution, it is important for authors to build their work on the existing theory and extend it, or to develop new theory. Quantitative papers should build their hypotheses based on an underlying theory. Qualitative papers usually develop research questions based on theory and/or develop new theory. No matter which approach you choose, it is vital to integrate the underlying theory properly in your manuscript. Merely citing a theory does not justify a theoretical contribution-it does not even pass as a sound theoretical basis. We recommend authors to clearly articulate their theoretical contributions briefly at the end of the introduction and to elaborate on theoretical advancements in detail in the discussion.

\section{Writing}

While quantitative papers follow a standard structure, qualitative and conceptual papers have more variety in structures. Papers in ABM follow typical structures of articles published in management journals. These standards are firmly established. For instance, quantitative papers have typically the following structure: (1) introduction, (2) theory, (3) methods, (4) results, and (5) discussion. If you fail to structure your work accordingly, the paper is likely to get desk-rejected. Language and spelling is another writing-related concern of some submissions-not only for non-native English authors. Furthermore, journals have different style guides, and many manuscripts do not comply with the ABM style guide or use inconsistent formatting. 
To increase readability and professionalism, authors are recommended to follow common standards of management research manuscripts (see recent articles in ABM). Authors should carefully check their manuscript and/or hire a professional proofreader prior to journal submission. Furthermore, ABM's style guide can be found here: https://www.palgrave.com/gp/journal/41291/authors/presentation-forma tting. The style guide also includes a recommendation regarding the length of articles. ABM recommends 7000-10,000 words. We believe that shorter articles do not allow for sufficient depth, while longer articles usually lack focus. If authors submit to ABM, they are expected to adhere fully to ABM's style guide.

\section{Methods}

Methodological rigor is important for quantitative and qualitative studies. Methodological rigor will be scrutinized in detail during the peer-review process. At the initial desk-review stage, we screen for major methodological flaws. One such major concern is small sample size. While we do not have clear cut-off sample sizes, as this would vary substantially depending on employed research methods, we do expect sufficiently large sample sizes to infer generalizable results. Quantitative studies need sufficient statistical power. Qualitative studies need sufficient data to generate reliable findings. Another major concern is whether methods are suitable to tackle corresponding research questions and if the methods are applied appropriately. In this regard, we frequently observe inappropriate statistical analyses, problematic operationalization of variables, and ecological fallacy. Unclear and incomplete description of samples, procedures, and analyses also raise concerns.

It is important for submitting authors to employ the appropriate methods to answer their research questions. Given the variety of different methods, it is difficult to propose specific recommendations. Rather, we recommend inexperienced researchers to receive guidance from methods experts to select and implement the adequate methods in a rigorous manner. If authors use standard methods, e.g., OLS regressions, standard reporting is sufficient. If authors use qualitative or less-common quantitative methods, it is essential that authors elaborate more on the methods applied so that editors and reviewers can clearly assess the appropriateness and quality.

\section{Concluding remarks}

It is important for us in our role as editors to stress that it is our sincere wish to help authors improve and publish their work. We do not enjoy sending out desk-reject letters; on the contrary, we prefer to help authors develop their work. Therefore, in this editorial, it was our aim to highlight common pitfalls and provide specific recommendations to help authors improve their submission and survive the desk-review. While some of the problems can easily be rectified, e.g., style guide, others are more fundamental, e.g., lack of theoretical contribution, or inadequate methods. Therefore, we recommend authors to take extra time to ensure they achieve high scientific 
standards. As we know how much time authors spend on their research, we hope that this editorial will motivate them to carefully plan and conduct their research to avoid fundamental problems, select the right target journal, and then adapt their manuscript to conform to the respective guidelines in order to increase publication chances. We hope to receive more submissions to ABM that will pass the deskreview, further improve them during the review process, and publish them. We look forward to your valuable submissions.

\section{Reference}

Froese, F. J. (2018). Editorial. Asian Business \& Management, 17(1), 1-3.

Publisher's Note Springer Nature remains neutral with regard to jurisdictional claims in published maps and institutional affiliations. 